\title{
Synthesis, Characterization and Antibacterial Activity from Mixed Ligand Complexes of 8-Hydroxyquinoline and Tributylphosphine for Some Metal Ions
}

\begin{abstract}
RANA ABDULILAH ABBAS ${ }^{1}$, AMER J J ARAD ${ }^{2}$, ION MARIUS NAFLIU3 ${ }^{3}$, AURELIA CRISTINA NECHIFOR ${ }^{3 *}$
'Department of Chemical Industrial, Institute of Technology/ Baghdad, Middle Technical University, Iraq 2Department of Chemistry, College of Education for Pure Science /lbn-Al-Haitham, University of Baghdad 3Politehnica University of Bucharest, Faculty of Applied Chemistry and Materials Science, Department of Analytical Chemistry and Environmental Engineering, 1-7 Gh. P. Polizu Str., 011061, Bucharest, Romania.

$\mathrm{CO}^{+2}, \mathrm{Ni}^{+2}, \mathrm{Cu}^{+2}$ as well $\mathrm{Zn}^{+2}$ compounds mixed ligand from 8-hydroxyquinoline(8-HQ) also tributylphosphine $\left(\mathrm{PBu}_{3}\right)$ have been attended at aquatic ethyl alcohol for (1:2:2) (M:8-HQ:PBu$)_{3}$. Produced complexes have been identified by utilizing atomic absorption flame, FT-IR as well UV-Vis spectrum manners also magnetic susceptibility as well as conductivity methods. At addendum antibacterial efficiency from the ligands as well complexes oboist three species about bacteria have been as well examined. Ligands and their complexes show good bacterial efficiencies. Of the gained datum the octahedral geometry was proposed into whole prepared complexes
\end{abstract}

Keywords: Mixed ligand, complexes, 8-hyroxyquinoline, tributylphosphine

8-Hydroxyquinoline has been reacted with many metal ions to form five stable membrane ring, it was chelated with metal ion by the oxygen atom after replaceable the hydrogen and heterocyclic nitrogen atom [1]. It has been found antibacterial activity and it used for determination of metal ions [2,3]. 8-hydroxyquinoline and their derivatives have been reported bioactivities containing anticancer, antibacterial, antidyslipidemic and antioxidative properties [4]. Mixed ligand complexes containing 8-hydroxyguinoline have been played an important area in the enzymes activation [5], these complexes have been appeared microbial activity against pathogenic microorganisms. Therefore, metal complexes which contain 8-hydroxyquinoline as ligand show biological activity [6]. The current paper refers to preparation of some metal complexes with mixed ligand from 8-hydroxyquinoline and tributylphosphine.

\section{Experimental part}

Instrumentation and materials

UV-Vis spectrum have been registered at a (Shimadzu UV-160 A) Ultra Violet-Visible Spectrophotometer. I.Rspectra were possessed at a (Shimadzu, FTIR-8400 S) Fourier Transform Infrared Spectrophotometer (4000-400) $\mathrm{cm}^{-1}$. Atomic Absorption was acquired through employing a(Shimadzu A.A-160A) Atomic Absorption/ Flame Emission Spectrophotometer. Conductors of $10^{-3} \mathrm{M}$ from complexes measured at DMSO on $25^{\circ} \mathrm{C}$ through employing (Philips PW- Digital Conduct meter). Magnetic characteristics were perfect through utilizing Auto Magnetic Susceptibility Balance Sherwood Scientific apparatus in $25 \mathrm{C}^{\circ}$. moreover, melting points were gained through employing (Melting Point Apparatus).

The following chemicals have been utilized like received of providers; cobalt chloride hexahydrate, nickel chloride hexahydrate, copper chloride dihydrate, zinc chloride (Merck), 8-hydroxyquinoline as well tributylphosphine (B.D.H).
Study of bacterial activity

The ligands and their metal complexes were tested versus bacteria: Staphylococcus aureus, E-coli, Pseudomonas employing the paper disc mode. Ready compounds at DMSO solution have been utilized at vitro through paper disc mode. Every materials utilized have been steriled at a hot air oven as well settlement from each to the screened micro organisms have been subcultured also brood around ( $8 \mathrm{~h}$ ) before introducing to the agar plates. The discs ( $7 \mathrm{~mm}$ diameter) have been soaked for different exam samples (condensation 1000ug/ $\mathrm{mL}$ ) drained as well thereafter placed at agar plate utilizing steriled tongs. Plates have been brood at $37^{\circ} \mathrm{C}$ with $(48 \mathrm{~h})$, at the end from nursery period; Zones from inhibition tothe various bacteria have been completed [7-9] .

\section{Preparation of Metal Complexes}

An aqueous solution at metal salts including $0.118 \mathrm{~g}$ $0.118 \mathrm{~g}, 0.085 \mathrm{~g}$ also $0.068 \mathrm{~g}$ ( $1 \mathrm{mmole}$ ) from cobalt chloride hexahydrate, nickel chloride hexahydrate, copper chloride dehydrate as well zinc chloride sequences has been added progressively for stirring to ethyl alcohol $\mathrm{NaOH}$ solution $(0.140 \mathrm{~g}, 2 \mathrm{mmol})$ of 8 -hydroxyquinoline. ( $0.5 \mathrm{~mL}, 2 \mathrm{mmole})$ of tributylphosphine $\left(\mathrm{PBu}_{3}\right)$ was added into mix on the every status through employing stoichiometric amount (1:2:2) Metal:8-HQ:PBu molar ratio. Mix has been refluxed for continous stirring about an hour. Mix has been cooled in room temperature dark precipitate was created, filtered and recrystallized of ethanol, the following reaction according of scheme 1.

\section{Result and discussions}

8-hydroxyquinoline ligand (8-HQ) has been specified through FT.IR as well UV-Vis. The solid compounds were ready by reaction from ethyl alcohol solution of the ligand for aqueous solution from metal ions and tributylphosphine into a (M:8-HQ:PBu$)$ from (1:2:2). Metal contents from complexes were into good correspondence with calculated values (table1) contains physical characteristics. Molar conductance from compounds like $\left(10^{-3} \mathrm{M}\right)$ on DMSO 
2<smiles>Oc1cccc2cccnc12</smiles>

2<smiles></smiles>

Scheme 1. The expected geometry of the metal(II) complexes $\left[\mathrm{M}(\mathrm{HQ})_{2}\left(\mathrm{PBu}_{3}\right)_{2}\right]$

Table 1

PHYSICAL CHARACTERISTICS INTO LIGAND AS WELL COMPLEXES

\begin{tabular}{|c|c|c|c|c|c|c|}
\hline Compounds & Color & M.PC & Yield\% & $M \%$ & 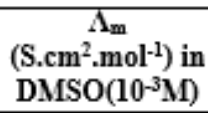 & $\begin{array}{c}\mu_{\text {eff }} \\
(B . M)\end{array}$ \\
\hline Ligand(8-HQ) & white & 74 & - & - & - & - \\
\hline$\left[\mathrm{Co}(8-\mathrm{HQ})_{2}(\mathrm{PBu})_{2}\right)^{2}$ & brown & $212-215$ & 77 & $\begin{array}{c}8.66 \\
(7.88)\end{array}$ & 10.45 & 4.84 \\
\hline$\left[\mathrm{Ni}(8-\mathrm{HQ})_{2}(\mathrm{PBu})_{2}\right]$ & green & $220-222$ & 75 & $\begin{array}{c}8.62 \\
(7.79)\end{array}$ & 12.64 & 2.90 \\
\hline$\left[\mathrm{Cu}(8-\mathrm{HQ})_{2}\left(\mathrm{PBu}_{3}\right)_{2}\right]$ & dark blue & $232-234$ & 71 & $\begin{array}{c}9.33 \\
(8.93)\end{array}$ & 14.77 & 1.72 \\
\hline$\left[\mathrm{Zn}(\mathrm{8}-\mathrm{HQ})_{2}\left(\mathrm{PBu}_{3}\right)_{2}\right]$ & pal yellow & $227-279$ & 75 & $\begin{array}{c}9.46 \\
(8.85)\end{array}$ & 15.33 & Dia \\
\hline
\end{tabular}

Table 2

THE FUNDAMENTAL FREQUENCIES TO THE LIGANDS AND THEIR COMPOUNDS $\left(\mathrm{cm}^{-1}\right)$

\begin{tabular}{|c|c|c|c|c|c|c|}
\hline Compounds & $\mathrm{v}(\mathrm{OH})$ & $v(\mathrm{C}=\mathrm{N})$ & $v(C=C)$ & $v(M-N)$ & $v(M-0)$ & $v(M-P)$ \\
\hline Ligand(8-HQ) & $3180 \mathrm{br}$. & 1624 sho. & $\begin{array}{l}1577 \mathrm{sh} \\
1504 \mathrm{sh} .\end{array}$ & - & - & - \\
\hline$\left[\mathrm{Co}\left(8-\mathrm{HQ}_{2}\left(\mathrm{PBu}_{3}\right)_{2}\right]\right.$ & - & $1639 \mathrm{~s}$. & $\begin{array}{l}1577 \mathrm{sh} \\
1500 \mathrm{sh}\end{array}$ & $513 \mathrm{w}$ & $474 \mathrm{w}$. & $435 \mathrm{w}$. \\
\hline$\left[\mathrm{Ni}\left(8-\mathrm{HQ}_{2}\left(\mathrm{PBu}_{3}\right)_{2}\right]\right.$ & - & $1643 \mathrm{~s}$. & $\begin{array}{l}1577 \mathrm{~s} . \\
1508 \mathrm{~s} .\end{array}$ & $513 \mathrm{w}$. & $474 \mathrm{w}$. & $450 \mathrm{w}$. \\
\hline$\left[\mathrm{Cu}\left(8-\mathrm{HQ}_{2}\left(\mathrm{PBu}_{3}\right)_{2}\right]\right.$ & - & $1638 \mathrm{~s}$. & $\begin{array}{l}1600 \mathrm{~s} . \\
1500 \mathrm{sh} .\end{array}$ & $520 \mathrm{w}$. & $466 \mathrm{w}$. & $439 \mathrm{w}$. \\
\hline$\left[\mathrm{Zn}(8-\mathrm{HQ})_{2}\left(\mathrm{PBu}_{3}\right)_{2}\right]$ & - & $1639 \mathrm{~s}$. & $\begin{array}{l}1577 \mathrm{~s} . \\
1500 \mathrm{~s} .\end{array}$ & $474 \mathrm{w}$. & $455 \mathrm{w}$. & $447 \mathrm{w}$. \\
\hline
\end{tabular}

sho=shoulder, $s=$ strong, sh=sharp, $w=w e a k, b r=$ broad

consist of non-electrolytic style [10], the datum were tabulat at (table 1). The magnetic moments (table 1) from complexes lie at range (1.72-4.84) B.M. That value indicates to a paramagnetic (high spin) whom was reported with most octahedral structure. At case from Zn(II) complex due of the $d$-orbital filled, subsequently the magnetic moment $(\mu=0)$ is diamagnetic [11].

\section{IR-Spectra}

Vibration bands for ligands and their compounds were registered into $\mathrm{KBr}$ at the area $4000-400 \mathrm{~cm}^{-1}$. The assignments to the distinctive bands (FT-IR) spectrum to 8-HQ, $\mathrm{PBu}_{3}$ and compounds are abbreviated at table 2. IR spectral for ligand (8-HQ) (fig.1) show wide band in 3180 $\mathrm{cm}^{-1}$, whom has been qualified into stretching vibration for $v(\mathrm{OH})$ phenol, this band was vanish into the spectra (fig.2) for all generated complexes, whom referred coordination this band for metal ion [12]. Band in1624 $\mathrm{cm}^{-1}$ appointed into $v(C=N)$ stretching frequency [13], on complexation a shifting for alteration into form were noticed of these bands, whereas growing into density were observe, specified may be a outcome from coordination for metal ion. Bands into IR spectrum to the 8-HQ in 1577 and 1504 $\mathrm{cm}^{-1}$ consequent into stretching vibration for $\mathrm{v}(\mathrm{C}=\mathrm{C})$ [14]. New bands watched in (435-513) $\mathrm{cm}^{-1}$ are temporarily appointed into $v(M-N), v(M-O)$ as well $v(M-P)$ (MetalLigands) stretching bands $[15,16]$. 


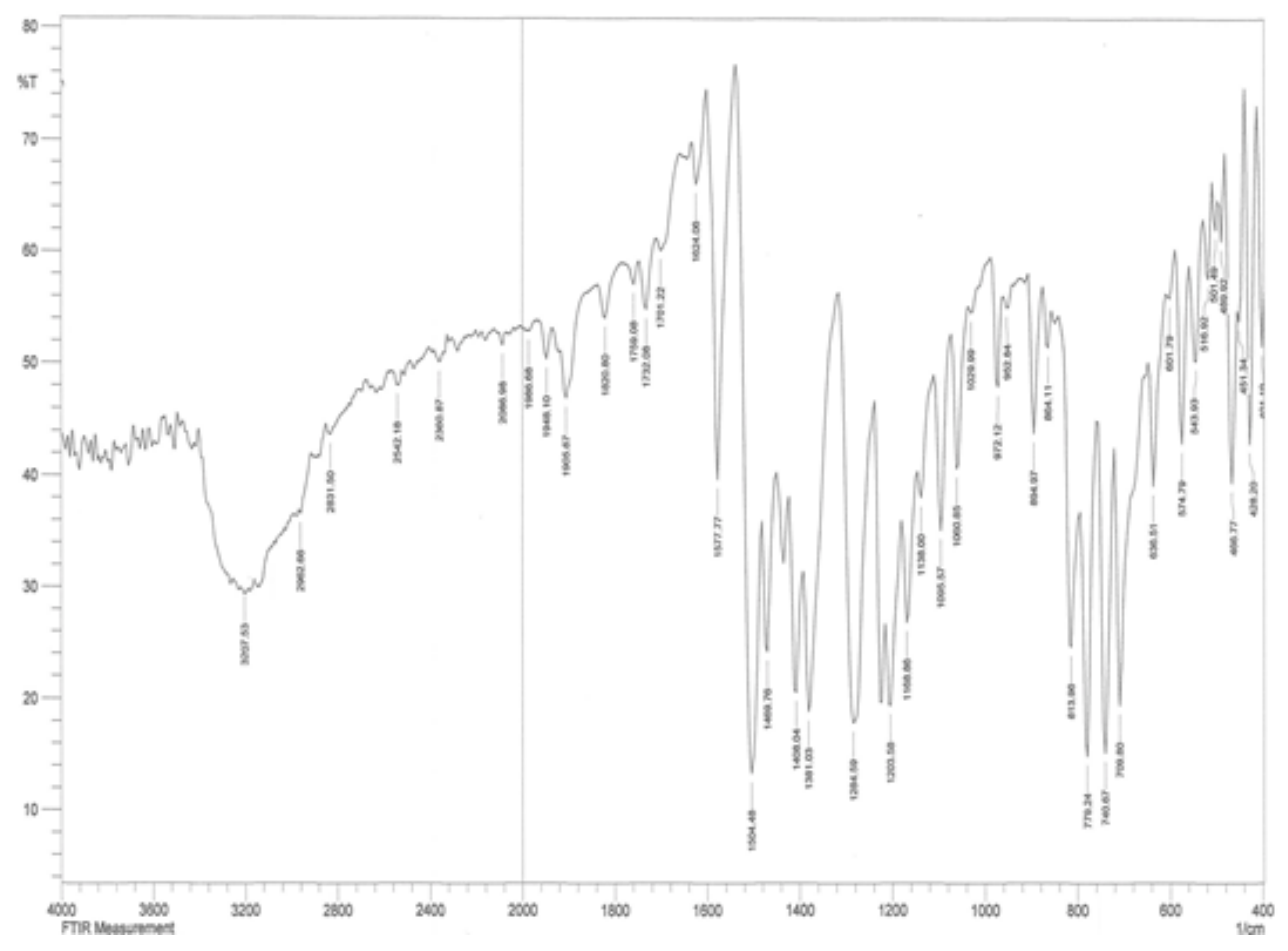

Fig.1. FTIR spectrum of 8hydroxyquinoline.

甲 SHIMADZU

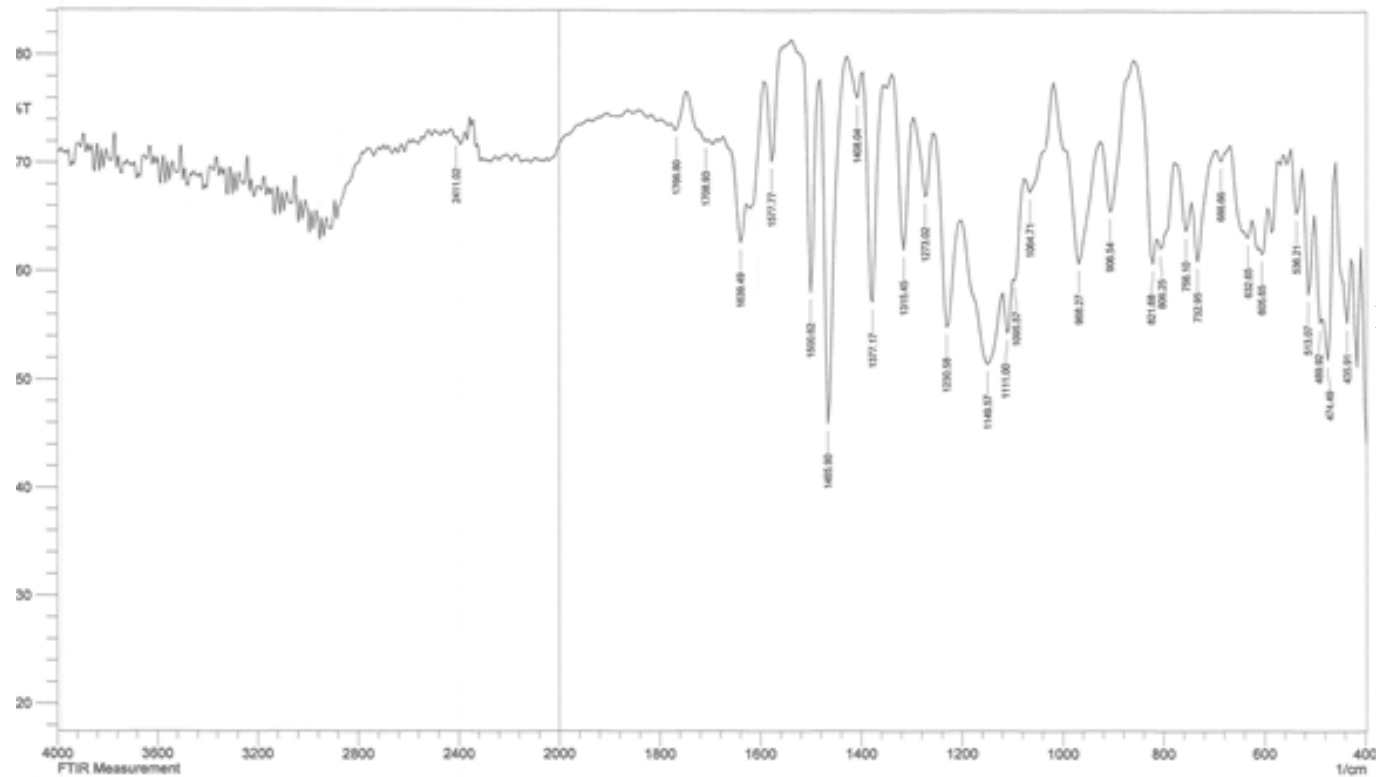

Fig. 2. FTIR spectrum of [Co(8$\left.\mathrm{HQ})_{2}\left(\mathrm{PBu}_{3}\right)_{2}\right]$ complex.

\section{UV-Vis spectra}

UV-Vis spectrum with ligands as well their compounds are included at (table 3). Spectrum of 8-HQ (fig.3) displays two peaks in 332 and $372 \mathrm{~nm}$ were appointed into $\left(\pi-\pi^{*}\right)$ [17]. The $\mathrm{PBu}_{3}$ spectrum exhibit absorption peak in 297 $\mathrm{nm}$ was qualified into $\left(\pi-\pi^{*}\right)$ [18]. Spectrum for $\mathrm{Co}^{+2}$ complex displays peaks, in 308 as well $398 \mathrm{~nm}$ were qualified into ligand field and charge transfer. Three peaks in 658, 732 and $920 \mathrm{~nm}$ were referred into electronic

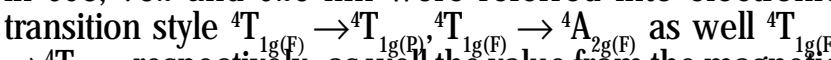
$\rightarrow{ }^{4} \mathrm{~T}_{2 \mathrm{f}(\mathrm{F})}$ respectively, as well the value from the magnetic moment in 4.84 B.M may be possessed as extra confirmation to octahedral geometry [19] . The electronic spectrum for $\mathrm{Ni}^{+2}$ complex gave two peaks, in 336 and $336 \mathrm{~nm}$ consequent into ligand field. Another three peaks in 537,722 and $830 \mathrm{~nm}$ were appointed into electronic transition style ${ }^{3} \mathrm{~A}_{2 \mathrm{~g}} \rightarrow{ }^{3} \mathrm{~T}_{1 \mathrm{~g}(\mathrm{P},}{ }^{3} \mathrm{~A}_{2 \mathrm{~g}} \rightarrow{ }^{3} \mathrm{~T}_{1 \mathrm{~g}(\mathrm{~F})}$ as well ${ }^{3} \mathrm{~A}_{2 \mathrm{~g}} \rightarrow$ ${ }^{3} T_{20(F)}$ continuity. Magnetic moment for this complex in 2.90 B.M whom was much close to the octahedral perimeter [20]. The spectrum for $\mathrm{Cu}^{+2}$ complex (fig.4) appears three peaks, the first also second at 308 as well $398 \mathrm{~nm}$ due to ligand field and charge transfer, the third pea seems in $658 \mathrm{~nm}$ was qualified into electronic transition style ${ }^{2} \mathrm{E} \rightarrow{ }^{2} \mathrm{~T}$. The magnetic moment for this complex was found in 1.72 B.M was much close to the octahedral environment [21]. Electronic spectral from $\mathrm{Zn}^{+2}$ complex do offer charge transfer, and the magnetic susceptibility seemed the complex has diamagnetic moments, resultto $d$-d transition are not likely subsequently electronic spectrum did not confer any productive datum, on fact this outcome is a good agreement for former work from octahedral geometry [22]. Antibacterial activity of the ligands and their compounds have as well been examined oboist choice chosen species for bacteria, (table $4)$. Depend on the consequences gained and spectral analysis an octahedral geometry has been proposed of produced compounds. 


\begin{tabular}{|c|c|c|c|c|c|}
\hline Compounds & $\begin{array}{l}\lambda_{\max } \\
(\mathrm{nm})\end{array}$ & ABS & $\begin{array}{c}\text { Wave } \\
\text { number } \\
\left(\mathrm{cm}^{-1}\right)\end{array}$ & $\begin{array}{c}€_{\max } \\
\left(\mathrm{L} \cdot \mathrm{mol}^{-1} \cdot \mathrm{cm}^{-1}\right)\end{array}$ & Remarks \\
\hline Ligand(8-HQ) & $\begin{array}{l}332 \\
372\end{array}$ & $\begin{array}{l}0.819 \\
0.861\end{array}$ & $\begin{array}{l}30120 \\
26881\end{array}$ & $\begin{array}{l}819 \\
861\end{array}$ & $\begin{array}{l}\left(\pi-\pi^{*}\right) \\
\left(\pi-\pi^{*}\right)\end{array}$ \\
\hline$\left[\mathrm{Co}(\mathrm{8}-\mathrm{HQ})_{2}\left(\mathrm{PBu}_{3}\right)_{2}\right]$ & $\begin{array}{l}308 \\
398 \\
658 \\
732 \\
920\end{array}$ & $\begin{array}{l}0.606 \\
0.645 \\
0.053 \\
0.042 \\
0.022\end{array}$ & $\begin{array}{l}32467 \\
25125 \\
15384 \\
13661 \\
10869 \\
\end{array}$ & $\begin{array}{l}606 \\
645 \\
53 \\
42 \\
22\end{array}$ & $\begin{array}{c}\text { L.F } \\
\text { C.T } \\
{ }^{4} \mathrm{~T}_{\lg (\mathrm{I}) \rightarrow} \rightarrow^{4} \mathrm{~T}_{\lg (\mathrm{P})} \\
{ }^{4} \mathrm{~T}_{\mathrm{lg}_{\mathrm{g}}(\mathrm{F}) \rightarrow{ }^{4} \mathrm{~A}_{2 \mathrm{~g}(\mathrm{f})}} \\
{ }^{4} \mathrm{~T}_{\mathrm{lg}_{\mathrm{g}(\mathrm{F})} \rightarrow{ }^{4} \mathrm{~T}_{2 \mathrm{~g}(\mathrm{~F})}}\end{array}$ \\
\hline$\left[\mathrm{Ni}(8-\mathrm{HQ})_{2}\left(\mathrm{PBu}_{3}\right)_{2}\right]$ & $\begin{array}{l}336 \\
376 \\
537 \\
722 \\
830\end{array}$ & $\begin{array}{l}0.980 \\
1.028 \\
0.028 \\
0.013 \\
0.011\end{array}$ & $\begin{array}{l}29761 \\
26595 \\
18621 \\
13850 \\
12048\end{array}$ & $\begin{array}{c}980 \\
1028 \\
28 \\
13 \\
11\end{array}$ & $\begin{array}{c}\text { L.F } \\
\text { L.F } \\
{ }^{3} \mathrm{~A}_{2 \mathrm{~g}(\mathrm{~F})} \rightarrow{ }^{3} \mathrm{~T}_{1 \mathrm{~g}(\mathrm{P})} \\
\left.{ }^{3} \mathrm{~A}_{2 \mathrm{~g}(\mathrm{P})} \rightarrow{ }^{3} \mathrm{~T}_{1 \mathrm{~g}(\mathrm{~F})}\right) \\
{ }^{3} \mathrm{~A}_{2 \mathrm{~g}(\mathrm{P})} \rightarrow{ }^{3} \mathrm{~T}_{2 \mathrm{~g}(\mathrm{~F})}\end{array}$ \\
\hline$\left[\mathrm{Cu}(\mathrm{8}-\mathrm{HQ})_{2}\left(\mathrm{PBu}_{3}\right)_{2}\right]$ & $\begin{array}{l}308 \\
398 \\
658\end{array}$ & $\begin{array}{l}0.606 \\
0.645 \\
0.053\end{array}$ & $\begin{array}{l}32467 \\
25125 \\
15197\end{array}$ & $\begin{array}{c}606 \\
645 \\
53\end{array}$ & $\begin{array}{c}\text { L.F } \\
\text { C.T } \\
{ }^{2} \mathrm{E}_{\mathrm{g}} \rightarrow{ }^{2} \mathrm{~T}_{2 \mathrm{~g}}\end{array}$ \\
\hline$\left[\mathrm{Zn}\left(8-\mathrm{HQ}_{2}\left(\mathrm{PBu}_{3}\right)_{2}\right]\right.$ & $\begin{array}{l}380 \\
391\end{array}$ & $\begin{array}{l}0.822 \\
0.937\end{array}$ & $\begin{array}{l}26315 \\
25575\end{array}$ & $\begin{array}{l}0.822 \\
937\end{array}$ & $\begin{array}{l}\text { C.T } \\
\text { C.T }\end{array}$ \\
\hline
\end{tabular}

Table 3

UV-Vis SPECTRUM DATUM

TO THE LIGANDS AND

THEIR COMPOUNDS

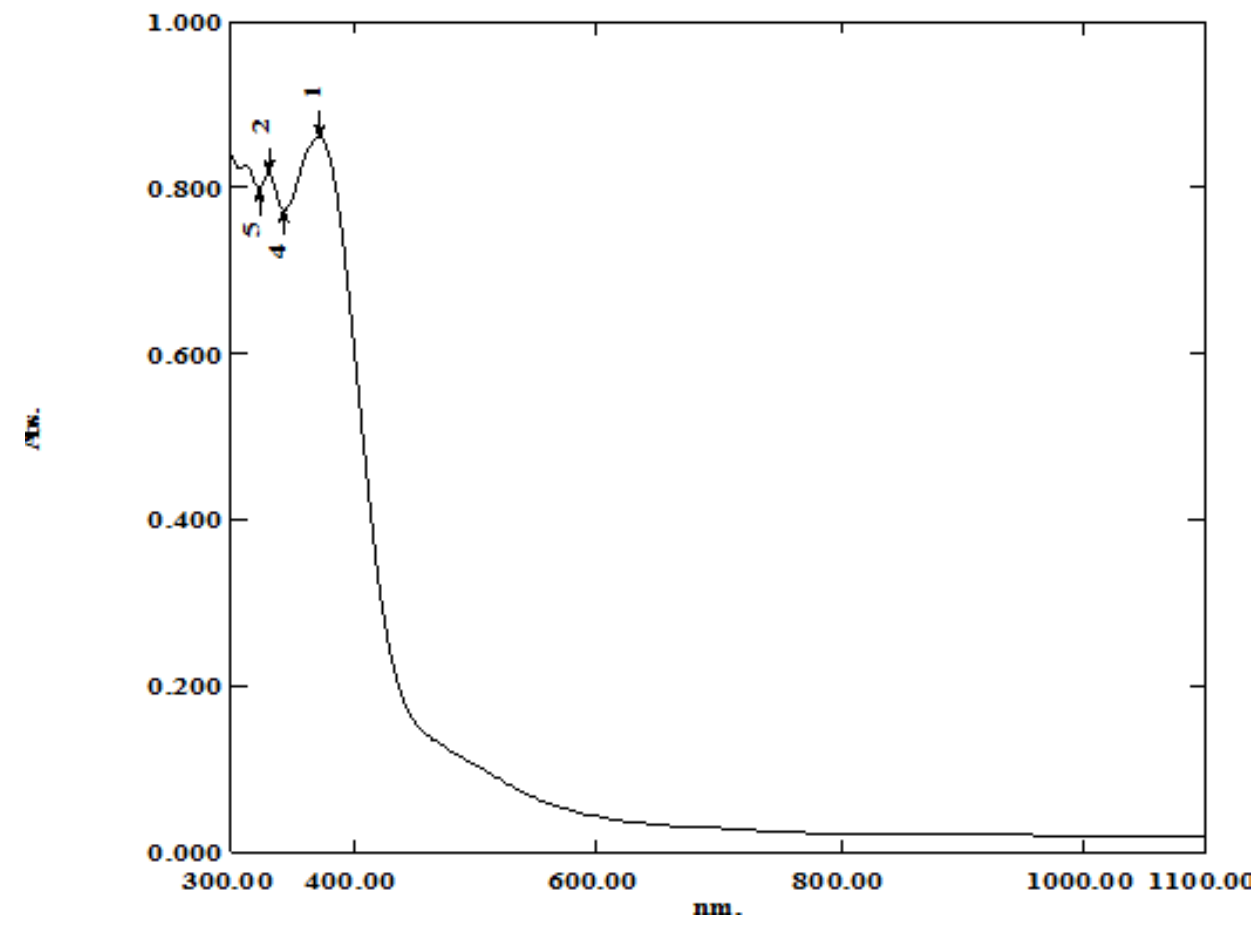

Fig. 3. UV-Vis spectrum of 8-hydroxyquinoline.

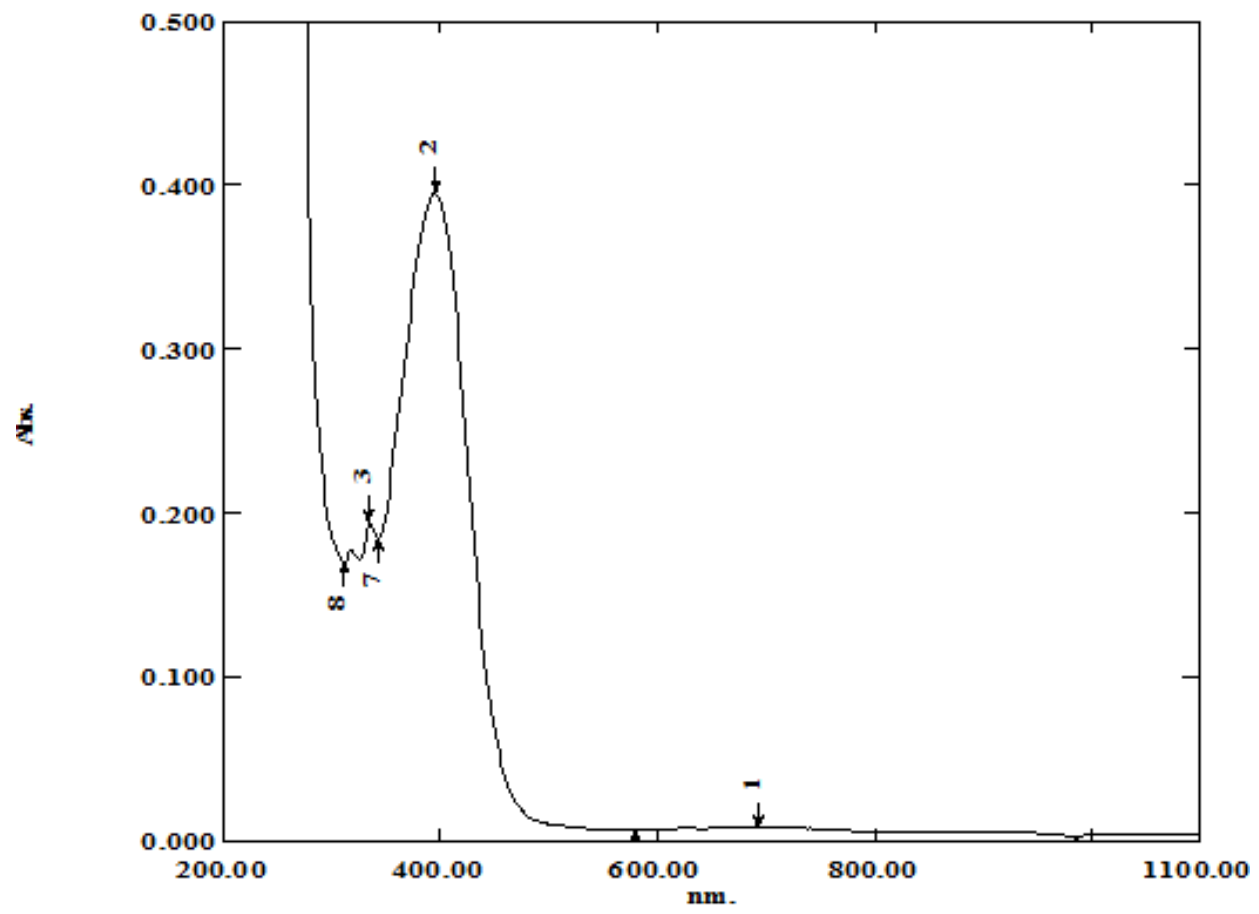

Fig. 4. UV-Vis spectrum of $\left[\mathrm{Cu}(8-\mathrm{HQ})_{2}\left(\mathrm{PBu}_{3}\right)_{2}\right]$ complex. 


\begin{tabular}{|c|c|c|c|}
\hline Compounds & $\begin{array}{c}\text { Staphylococcus } \\
\text { Aurous }\end{array}$ & $\begin{array}{c}\text { Escherichia } \\
\text { Col }\end{array}$ & Pseudomonas \\
\hline Ligand(8-HQ) & 22 & 25 & 25 \\
\hline $\mathrm{PBu}_{3}$ & 20 & 22 & 20 \\
\hline$\left[\mathrm{Co}\left(8-\mathrm{HQ}_{2}\left(\mathrm{PBu}_{3}\right)_{2}\right]\right.$ & 30 & 30 & 22 \\
\hline$\left[\mathrm{Ni}\left(8-\mathrm{HQ}_{2}\left(\mathrm{PBu}_{3}\right)_{2}\right]\right.$ & 26 & 22 & 20 \\
\hline$\left[\mathrm{Cu}\left(8-\mathrm{HQ}_{2}\left(\mathrm{PBu}_{3}\right)_{2}\right]\right.$ & 20 & 25 & 25 \\
\hline$\left[\mathrm{Zn}\left(8-\mathrm{HQ}_{2}\left(\mathrm{PBu}_{3}\right)_{2}\right]\right.$ & 20 & 20 & 20 \\
\hline
\end{tabular}

Table 4

DIAMETERS (mm) TO SUPPRESSION TO THE BACTERIA OF COMPOUNDS

\section{Conclusions}

Mixed ligand complexes may be a synthetic defy to tune the characteristics from metal complexes as well were offered to display a wide range of. The probable geometry from synthesis complexes is octahedral as well it is six coordinated ligand metal complexes.

\section{References}

1. SHAR, G.A., SOOMRO, G.A., J. Chem. Soc. Pak., 27, no.5, 2005, p. 471.

2. BOSCH, M.E., SANCHEZ, A.J .R., ROJ AS, F.S., OJEDA, C.B., J. Pharm. Biomed. Anal., 44, no. 4, 2007, p. 831.

3. AHMED, W., KHAN, S.A., MUNAWAR, K.S., KHALID, A., KAWANL, S., Tropical. J. Pharm. Res., 16, no.5, 2017, p. 1137.

4. RAJ, H.D., PATEL, Y.S., Advance Appl. Sci. Res., 6, no. 2, 2015, p. 119 5. THAKKAR, J.R., THAKKAR, N.V., Synthesis and Reactivity in Inorganic and Metal-Organic Chemistry, 3, no.10, 2000, p. 1871.

6. SHIVANKAR, V.S., THAKKAR, N.V., Acta. Poloniae Pharmaceutica, 60, no. 1, 2003, p. 45.

7. NITA, A., TIT, D. M., COPOLOVICI, L., MELINTE (FRUNZULICA), C. E., COPOLOVICI, D., BUNGAU, S., Rev. Chim.(Bucharest), 68, no. 6, 2017, p. 1170.

8. INIAMA, G.E., NFOR, E.N., OKON, E.D., IORKPILIGH, I.T., Inter. J. Sci. Techn. Res., 3, no. 11, 2014, p. 73.

9. BUNGAU, S., TIT, D. M., IOVAN, C., COPOLOVICl, L., BOSCENCU, R., CIOCA, G., COPOLOVICI, D., Rev. Chim.(Bucharest), 68, no. 11, 2017, p. 2495.
10. GEARY, W.J., Coord. Chem. Rev., 7, 1971, p. 81.

11. EL-GHAR, M.F., ABDEL-GHANI, N.T.,BADR, Y., EL-BORAADY, O.M., ISESCO. Science and Technology Vision, 3, 2007, p. 58.

12. J ARAD, A.J, KADHIM, Z.S., Int. J. Human. Arts. Med. Sci., 3, no. 10, 2015. 197-210.

13. JARAD, A.J., JAAFER, Z.N., ABDU-AMEER, N., Int. J. Sci. Res., 2, 2013, p. 64.

14. PALLIKAVIL, R., UMNATHUR, M.B., KRISHNANKUTY, K., Arch.Appl.Sci.Res., 4, 2012, p. 223.

15. JARAD, A.J., Eur. Chem. Bull., 2, no. 6, 2013, p. 383.

16. AL-NOOR, T.H., JARAD, A.J., HUSSEIN, A.O., SChem. Mat. Res., 6, no. 3, 2014, p. 20.

17. RAJ AVEL, R.,SENTHIL, M., ANITHA, C., E-J. Chem., 5, 2008, p. 620. 18. MOHAMED, G.G., OMAR, M.M., HINDY, A.N., Turk. J. Chem., 30, 2006, p. 61.

19. PATIL, A.J ., DONDE, K.J ., RAUT, S.S., PATIL, V.R., LOKHANDE, R.S., J. Chem. Pharm. Res., 4, no.2, 2012, p. 1413.

20. MAPARI, A.K., MANGAONKAR, K.V., Inter. J. Chem. Techn. Res., 3, no.1, 2011, p. 477.

21. SHRIODKAR, S.G., MANE, P.S., CHONDHEKAR, K., Indian J. Chem., 40A, 2001, p. 1114.

22. AL-NOOR, T.H., MANHEL, R.A., AL-TEBOORI, A.T., J. Chem. Mater. Res., 3, 2013, p. 114.

Manuscript received: 17.09 .2018 